\title{
Healthcare Cost of Smoking Induced Cardiovascular Disease in Tanzania
}

\author{
Asmerom Kidane ${ }^{1}$, Aloyce Hepelwa ${ }^{1}$, Ernest Tingum $\mathrm{Ngeh}^{1}$ and Teh-wei $\mathrm{Hu}^{2}$ \\ 1. Department of Economics, University of Dar es Salaam, Dar es Salaam POB 35045, Tanzania \\ 2. School of Public Health, University of California, Berkeley, California 94607, USA
}

Received: March 4, 2015 / Accepted: March 29, 2015 / Published: March 31, 2015.

\begin{abstract}
The study presented here estimates the total health care cost attributable to smoking induced cardiovascular disease in Tanzania. The study based on a survey conducted at a referral university hospital in Dar es Salaam in 2014. Assuming a 2\% prevalence rate of cardiovascular disease and a population of 47.2 million, it was estimated that there are 943,800 cardiovascular patients in Tanzania. The proportion of ever smokers among the surveyed patients was found to be 25 percent yielding 240,400 patients who suffer from smoking induced cardiovascular diseases. Per capita annual expenditure per patient is estimated to be 566.6 US dollars and total annual expenditure for the country was estimated to be 136.1 million US dollars. On a per capita basis more direct and indirect cost is incurred on males compared to females; more is spent on the elderly (40 or more years) compared to the youth (less than 20 years). When compared with the mean annual household income of the surveyed population, the smoking induced per capita expenditure constitutes $35 \%$ of household income.
\end{abstract}

Key words: Healthcare, cost, smoking, cardiovascular, Tanzania.

\section{Introduction}

Tanzania is one of the largest countries both in area $(26,000 \mathrm{sq} \mathrm{km})$ and a population of 47.2 million. The country is also one of the major producers of tobacco in the world and the second largest producer in Africa [1]. For the year 2011-2012, the area planted was 118,250 hectares with a yield of 126,620 tones giving 1.07 tons per hectare [2]. Export earned from tobacco for the same year was 252.6 million US dollars [2]. The latter constitutes $40 \%$ of total export earnings from traditional crops. With regards to cigarette smoking, the overall smoking prevalence rate is 12.4 for males, $8.8 \%$ for females and 10.6 overall [3-5]. The overall estimate increases to $20.5 \%$ when the reference population is limited to adults (15 to 60 years). Most smokers in Tanzania appear to lack of the understanding of the harmful health effects of smoking,

Corresponding author: Asmerom Kidane, Ph.D., research field: economic demographic modelling. E-mail: akidane@udsm.ac.tz. the corresponding higher health expenditure as well as reduced earnings that emanate from smoking related diseases.

Within Tanzanian agriculture tobacco leaf production is the most subsidized when compared with other basic necessities such as maize, rice and ground nut. The percent of tobacco farmers using improved seed, modern fertilizer and having access to credit facilities is $83.8,96.4$ and 61.7 percent respectively. The corresponding values for maize growers (the major staple diet of Tanzanians) are only 13.4, 34.6 and 9.1 percent respectively [6]. Most tobacco leaves are exported and some are used as inputs in the production of cigarette.

Cigarettes in Tanzania are produced by the National Tobacco Corporation. Government revenue from profit and excise tax are substantial. As a result, the government and parastatals appear to be reluctant to highlight the negative social and health impact of tobacco production and cigarette consumption. In 2007, 
the government of Tanzania ratified the WHO's Framework Convention on tobacco control. However implementing the convention guidelines appears to be lagging. Chances are that the revenue generated from cigarette production and consumption may be much less than the direct and indirect expenditure on health that emanate from tobacco production and cigarette smoking. There is the need to estimate the direct and indirect health care cost of smoking at a national level and compare the result with the revenue that accrues to the government from cigarette production and cigarette excise taxes.

At a household or at an individual level there are relatively few studies that consider the health care cost which emanates from smoking [7, 8]. In Tanzania there is hardly any detailed study related to estimating direct and indirect monetary expenditure on diseases emanating from cigarette smoking at an individual, at a regional or at a national level. The objective of the study is thus to provide a national estimate for the costs of smoking induced cardiovascular disease in Tanzania and compare the results with the excise tax revenue that accrues to the government.

\section{Methods}

The health care cost of smoking induced cardiovascular disease will be divided into direct, indirect as well as total cost. It should be noted that all cardiovascular diseases do not emanate from smoking and not all cardiovascular patients are smokers. An attempt is made to see the proportion of ever smokers from patients suffering cardiovascular diseases. This will enable us to isolate and estimate the total cost of treating cardiovascular diseases that are attributable to smoking. Assuming that survey results are representative of the Tanzanian population, total cost at a national level will also be estimated.

The data for the study are based on a 2014 survey that was conducted among patients with cardiovascular diseases. The questionnaire was administered in both China and Tanzania; the questionnaire was translated into Swahili- the official language of Tanzania. The survey was conducted at the Mihumbili University referral hospital located in Dar es Salaam Tanzania. 123 patients were interviewed over a period of two months. 59 patients were outpatients and 64 were inpatients. It should be noted that inpatients have serious cardiovascular problems when compared with outpatients. Enumerators included two doctors, a nurse, and two postgraduate students.

\section{Results and Discussion}

\subsection{Descriptive Statistics}

Before estimating costs the background characteristics of the respondents is estimated and presented in Table 1a. The results show that $46.6 \%$ of the outpatients as being males; the corresponding value for inpatients is $56.5 \%$. This result may be viewed with the fact that the intensity of smoking related diseases (inpatients) in Tanzania is much higher for males than females. The percent of males aged 40 years and above (the age range when tobacco related diseases are at their peak) is $66.1 \%$ for outpatients and $57.8 \%$ for inpatients. Compared to inpatients, outpatients seem to have smaller household size, higher monthly income and higher insurance coverage. The survey also shows that the percent of ever smokers among the cardiovascular patients as being $28.2 \%$ for outpatients and $21.9 \%$ for inpatients. When the percent of ever smokers among the surveyed population is cross classified by gender, male ever smokers make $41.3 \%$ of the respondents; the corresponding value for females is a mere 8.5 percent.

Table $1 \mathrm{~b}$ presents a distribution on the number of visits per year as classified by out and inpatients.

An assumption is made that the more frequent the visits by a patient, the more serious the disease. The results in Table $1 \mathrm{~b}$ show that $55.9 \%$ of outpatients have visited health centers at least three times per year; the corresponding value for inpatients is $40.3 \%$ suggesting that a large percent of the patients are suffering from serious cardiovascular ailment. When reclassified by 
Table 1 Descriptive statistics of the study population.

\begin{tabular}{llll}
\hline Variables & Outpatients & Inpatients & All \\
\hline Gender & & & \\
Males & $27(46.5)$ & $36(56.3)$ & $63(51.6)$ \\
Females & $31(53.5)$ & $28(43.7)$ & $59(48.4)$ \\
Age & & & \\
$<20$ & $6(10.7)$ & $4(6.3)$ & $10(8.1)$ \\
$20-39$ & $14(23.7)$ & $23(35.3)$ & $37(30.1)$ \\
$40-59$ & $23(39.0)$ & $19(29.7)$ & $42(34.2)$ \\
$\Rightarrow \quad 60$ & $16(27.1)$ & $18(28.1)$ & $34(27.6)$ \\
Household size & $5.04(2.6)^{*}$ & $5.61(2.9)^{*}$ & $5.33(2.8)^{*}$ \\
Monthly income** & $136.1(134.1)$ & $125.9(139.1)$ & $131.0((136.1)$ \\
Health insurance & $19(32.8)$ & $14(22.2)$ & $33(27.3)$ \\
Ever smoked $(\%)$ & $17(28.8)$ & $14(21.9)$ & $31(25.2)$ \\
Mean no. of years smoked*** & 9.9 & 20.9 & 14.9 \\
\hline
\end{tabular}

* values in parenthesis is standard deviation, $* *$ in US dollars, *** among ever smokers.

Table 1b Number of visits to health facility per year.

\begin{tabular}{|c|c|c|c|c|c|c|}
\hline \multirow{2}{*}{ Number of visits } & \multicolumn{2}{|c|}{ Outpatients } & \multicolumn{2}{|c|}{ Inpatients } & \multicolumn{2}{|c|}{ All } \\
\hline & Number & $\%$ & Number & $\%$ & Number & $\%$ \\
\hline 1 & 2 & 3.4 & 11 & 17.8 & 13 & 10.7 \\
\hline 2 & 24 & 40.7 & 26 & 41.9 & 50 & 41.3 \\
\hline 3 & 24 & 40.7 & 19 & 30.8 & 43 & 35.5 \\
\hline 4 & 9 & 15.2 & 5 & 8.2 & 14 & 11.7 \\
\hline 5 & 0 & 0 & 1 & 1.3 & 1 & 0.8 \\
\hline Total & 59 & 100 & 62 & 100 & 121 & 100 \\
\hline
\end{tabular}

gender, the percent of male and female inpatients that made three or more visits per year is $44.2 \%$ and $32.2 \%$ respectively. For outpatients the corresponding values are 48.2 and 64.5 percent.

\subsection{Estimating the Cost of Treatment of Cardiovascular Diseases}

In the following paragraphs an attempt is made to estimate the cost attributable to cardiovascular diseases among ever smokers. It is already noted that the costs are classified into two- - direct and indirect cost. Direct costs include hospital bills for both outpatients and inpatients along with the drug costs. Indirect costs include other costs related to hospital visitation, travel costs to and from the hospital, time lost by the patient (converted to monetary value) as well as the cost for a caregiver(s). The results, which are given as mean expenditure per patient, are summarized in Tables $2 \mathrm{a}-2 \mathrm{c}$.
The following observations may be drawn from Tables 2a-2c,

As expected, inpatient cost is $23.0 \%$ higher than outpatient's, direct cost is $55.0 \%$ higher than indirect cost.

When comparing on a per capita basis more direct and indirect cost is incurred on males compared to females. This result is consistent with the fact that the majority of the smokers are males; it should also be noted that males appear to have easier access to health facilities than females.

Among the tobacco induced cardiovascular patients, more expenditure is incurred on patients aged 40 to 59 years followed by those aged 20 to 39 years. Again this is consistent with similar studies elsewhere implying that prolonged smoking is more likely to increase the cost and prevalence of tobacco related diseases. Relatively less amount is spent among ever smokers aged less than 20 years. 
An attempt is also made to compare the healthcare cost per patient with per household annual income. For inpatients and outpatients total healthcare cost constitute $32 \%$ and $39 \%$ of annual income respectively. This is a major drain on the already low household income in Tanzania.

\subsection{Estimating the Cost of Smoking Attributable} Diseases for Tanzania

Based on the results of the stated survey, an attempt is made to estimate the aggregate cost of smoking attributable cardiovascular disease for the whole country. The following information will be needed:

(1) The age and gender distribution of ever smokers, this will be estimated from survey result;

(2) The size and gender distribution of Tanzanian population for 2013;

(3) The prevalence of cardiovascular diseases in 2013 for Tanzania.

\subsection{Size and Gender Distribution of Tanzanian Population}

It was earlier noted that the total population of Tanzania for 2013 was 47.2 million. Males constitute 49 percent while females are 51 percent The national prevalence rate of cardiovascular diseases is about 20 per 1,000 or 2 percent which gives us 943,000 cardiovascular patients (Tanzanian health professionals state that this is an underestimate). The age distribution of these patients is obtained from survey results. It should again be noted that not all cardiovascular diseases are due to smoking only. This leads one to estimating the proportion of ever smokers among the cardiovascular patients; this is again obtained from survey and is given in column 3 of Table 3 . The table also gives the total cardiovascular patients and the proportion of ever smokers among these patients; the age distribution is also given.

The results above show that there were about 240,400 smoking induced cardiovascular patients in Tanzania in 2013. The table also shows that the magnitude of smoking induced cardiovascular disease increases with age; more than 50 percent of cardiovascular patients aged 60 and above are classified as smoking induced.

\subsection{Estimated Cost of Tobacco Related Illness for Tanzania}

Based on the results in last column of Table $2 \mathrm{c}$ (cost estimate) and Table 3 column 4 (number of patients), an estimate of direct, indirect and the total annual cost of smoking in US dollars for Tanzania. The result is given in Table 4 . The age distribution of expenditure group is also provided.

The above results show that the total smoking related health expenditure in Tanzania was 136.1 million dollars per year. Sixty-one percent of this is direct while $39 \%$ is indirect. Most of the patients appear to be elderly who have been smoking for many years; this is likely to prolong recovery, increase health care costs,

Table 2a Annual total, direct and indirect costs of cardiovascular patients: by patient status.

\begin{tabular}{llll}
\hline Cost* & Outpatients & Inpatients & All patients \\
\hline Direct & $354.8(444.4)$ & $334.8(389.1)$ & $344.4(414.9)$ \\
Indirect & $150.9(543.3)$ & $288.3(594.5)$ & $222.2(572.4)$ \\
Total & $505.7(857.0)$ & $623.1(834.3)$ & $566.6(843.9)$ \\
\hline
\end{tabular}

* In US dollars; 1 US dollar = 1620 Tanzania shilling; value in parenthesis are standard deviation.

Table 2b Annual total, direct and indirect costs of cardiovascular patients: by gender.

\begin{tabular}{llll}
\hline Cost & Males & Female & All patients \\
\hline Direct & $387.5(526)$ & $300.3(249.4)$ & $344.4(414.9)$ \\
Indirect & $261.3(678.0)$ & $140.1(302.1)$ & $222.2(572.4)$ \\
Total & $648.8(1052.7)$ & $440.4(448)$ & $566.6(843.9)$ \\
\hline
\end{tabular}

* In US dollars. 
Table 2c Annual total, direct and indirect costs of cardiovascular patients: by age and gender*.

\begin{tabular}{llll}
\hline Age & Direct & Indirect & All patients \\
\hline$<20$ & $333(197)$ & $159(433)$ & $492(598)$ \\
$20-39$ & $299(308)$ & $264(541)$ & $552(240)$ \\
$40-59$ & $471(598)$ & $245(683)$ & $716(1128)$ \\
$60-$ & $253(212)$ & $167(504)$ & $420(551)$ \\
Overall & $344.4(414)$ & $222.2(572)$ & $566.6(844)$ \\
\hline
\end{tabular}

* In US dollars.

Table 3 Estimated total and smoking induced cardiovascular patients classified by age.

\begin{tabular}{llll}
\hline Age & $\begin{array}{l}\text { Total cardiovascular patients* } \\
(1)\end{array}$ & $\begin{array}{l}\text { Proportion of smoking induced } \\
\text { cardiovascular patients }(2)\end{array}$ & $\begin{array}{l}\text { Total smoking induced } \\
\text { cardiovascular patients* }(3)=1 \times 2\end{array}$ \\
\hline Less than 20 & 75.5 & 0.10 & 7.6 \\
$20-39$ & 283.2 & 0.22 & 62.3 \\
$40-59$ & 320.9 & 0.10 & 30.5 \\
$60-$ & 264.3 & 0.53 & 140.1 \\
Total & 943.8 & 0.25 & 240.4 \\
\hline
\end{tabular}

* In thousands.

Table 4 Annual direct, indirect and total costs of smoke related cardiovascular diseases*.

\begin{tabular}{llll}
\hline Age & Direct & Indirect & Total \\
\hline$<20$ & 2.5 & 1.2 & 3.7 \\
$20-39$ & 18.0 & 16.0 & 34.0 \\
$40-59$ & 14.4 & 7.5 & 21.9 \\
$60-$ & 35.4 & 23.4 & 58.8 \\
Overall & 82.7 & 53.4 & 136.1 \\
\hline
\end{tabular}

* In million US dollars.

accelerate mortality and decrease life expectation. The total amount of earning from tobacco export for Tanzania is 335.4 million dollars while cigarette tax revenue is 54 million dollars (URT 2013) [2]. This suggests that revenue generated from cigarette tax is less than the expenditure on smoking induced cardiovascular diseases.

It is already noted that the above estimate is based on the assumption that the prevalence of cardiovascular disease in Tanzania is $2 \%$ which may be an underestimate. Tanzania is an African country with understaffed and inefficient health facilities, with per capita income of 560 dollars per year; the latter is three times lower than that of China. Access to health care centers is thus limited. In other words, the total cost may be an underestimate. Other reasons for the under estimation include the fact that many Tanzanian smokers belong to low income group and are less likely to have access to referral clinics; in other words, there may be a serious under reporting. Other smoke related diseases such as hip fracture, gum diseases are not considered. The loss in earnings due to higher mortality (low life expectation) is also not accounted for. Most important passive smoking is not included. The latter is likely to be high within a household setting where the mean household size is more than five individuals.

\section{Conclusion}

The prevalence of cigarette smoking in Tanzania is 10.6 percent - a relatively low value by world standards. However, the estimated total cost of smoking related cardiovascular disease is relatively high when compared with the country's overall or per capita GDP. A large percent of cigarette (raw tobacco) smokers in Tanzania belong to low income group and 
expenditure on smoking attributable diseases constitute a significant percent of household income. The mean monthly income between non smoking and ever smoking cardiovascular patients is compared; the survey results reveal that non smoking patients income was 138.2 US dollars; while the corresponding value for ever smoking patients 110.2 US dollars-a difference of $25.4 \%$. Besides, about $29 \%$ of non smoking cardiovascular patients appear to be insured; the corresponding value for ever smoking patients is only $23 \%$. In other words, the burden of health care cost is high among ever smokers.

It was also noted that the total national expenditure on smoking related cardiovascular cancer patients is an underestimate. A more comprehensive study on the health care costs of smoking induced diseases is likely to yield a higher cost. Policy makers in Tanzania ought to introduce policies and guidelines that ought to decrease the prevalence of ever smoking in Tanzania. This will help reduce the cost of smoking related diseases.

\section{Contributions}

Each author has contributed equally from early stages of survey to the final write-up of the document.

\section{Conflict of Interest}

There is no conflict of interest

\section{Funding}

This study was supported by a grant from United States National Institutes of Health-Fogarty
International Centre and National Cancer Institute R01 TW009295

\section{Conference Presentation}

The paper has been presented in several workshops and on the basis of this; improvement have been made.

\section{Reference}

[1] Kidane, A., Hepelwa, A., Ngeh, E. T., and Hu, T. W. 2015. "A Comparative Analysis of Technical Efficiency of Smallholder Tobacco and Maize Farmers in Tabora, Tanzania." Journal of Development and Agricultural Economics 7 (2): $72-9$.

[2] United Republic of Tanzania, 2014. Statistical Abstracts 2013.

[3] WHO, 2013. Report on the global tobacco epidemic, 2013, accessed December 15, 2014. www.who.int/iris/bitstream/10665/85380/1/97892415058 71_eng.pdf.

[4] Mundi Index, 2012. Accessed December 15, 2014. http://www.indexmundi.com/facts/indicators/SH.PRV.S MOK.FE/rankings.

[5] Kalage, R., Blomstedt, R., Preet, K., Hoffman M. B., and Kinsman, J. 2012. INTREC Tanzania Country Report

[6] Kidane, A., Hepelwa, A., Ngeh, E. T., and Hu, T. W. 2013. "Agricultural Input and Efficiency in Tanzania Small Scale Agriculture: A Comparative Analysis of Tobacco and Selected Food Crops." Tanzania Economic Review 3.

[7] Karki, Y., Pant, K., and Pande, B. 2013. A Study on the Economics of Tobacco in Nepal. Washington, DC: The World Bank.

[8] Sung, H. Y., Wang, L., Jin, S., Hu, T. W., and Jiang, Y. 2008. "Economic Burden of Smoking in China." In Tobacco Control Policy Analysis in China, edited by $\mathrm{Hu}$, T. W. New York: World Scientific. 